Check for updates

Cite this: RSC Adv., 2019, 9, 31186

Received 9th March 2019

Accepted 16th September 2019

DOI: 10.1039/c9ra01792e

rsc.li/rsc-advances

\section{Hybrid microfluidic sorting of rare cells based on high throughput inertial focusing and high accuracy acoustic manipulation $\uparrow$}

\begin{abstract}
Yinning Zhou, + Zhichao Mał and Ye Ai (DD *
The ability to isolate rare circulating tumor cells (CTCs) from blood samples is essential to perform liquid biopsy as a routine diagnostic and prognostic test. Both label-free and surface biomarker-based cell sorting technologies have been developed to address the demand in high-integrity isolation of rare CTCs for cancer research. Label-free cell sorting mainly relies on the size difference between CTCs and blood cells; thus, it lacks sufficient sorting specificity. Surface biomarker-based cell sorting is highly specific; however, it requires expensive, labor-intensive, and time-consuming labeling due to the use of multiple sets of surface biomarkers. Because of the complex nature and high heterogeneity of tumorigenesis, it is difficult to rely on a single sorting process for high-integrity rare cell isolation. In this study, for the first time, we present a hybrid microfluidic cell sorting method combining high throughput size-dependent inertial focusing for size-based pre-enrichment and high accuracy fluorescence activated acoustic sorting for single cell isolation. After one single hybrid sorting process, we have demonstrated at least 2500-fold purity enrichment of MCF-7 breast cancer cells spiked in diluted whole blood samples with cell viability maintained at $91 \pm 1 \%$ (viability before sorting was $94 \pm 2 \%$ ). This developed hybrid microfluidic cell sorting technique provides a promising solution for rare cell isolation needed in a variety of biological research and clinical applications.
\end{abstract}

\section{Introduction}

Cancer is one of the top causes of death around the world due to its lethality. ${ }^{1}$ One of the reasons why it is extremely difficult to achieve a radical cure of cancer is malignant metastasis, which is an overwhelming obstacle in therapeutic management and early diagnosis, and circulating tumor cells (CTCs) contribute to the very basis of this process. The concept of CTCs was first introduced by Ashworth in $1869,{ }^{2}$ but due to the extremely rare appearance of CTCs in the peripheral blood of patients (10-100 CTCs per $\mathrm{ml}^{3}$ ), the study of CTCs in cancer research started to gain increasing attention only in the past two decades when substantial advances were made in more reliable isolation and detection of low-abundance cell populations. ${ }^{4}$ Currently, the only CTC testing medical instrument clinically approved by US Food and Drug Administration (FDA) is CellSearch, which isolates CTCs via specific binding to magnetic nanoparticles functionalized with anti-EpCAM antibodies in a magnetic field. Although immune-affinity approaches possess the advantage of high specificity, the detection criteria of EpCAM, a unique

Pillar of Engineering Product Development, Singapore University of Technology and Design, Singapore 487372, Singapore. E-mail: aiye@sutd.edu.sg; Tel: +65 64994553 $\uparrow$ Electronic supplementary information (ESI) available. See DOI: 10.1039/c9ra01792e

\$ These authors contributed equally to this work. biomarker expressed during the epithelial-mesenchymal transition (EMT) process, ${ }^{5}$ will not necessarily express with all types of CTCs. ${ }^{6,7}$ Furthermore, leukocytes also exhibit high chances to express similar epithelial markers, which greatly increase the probability of erroneous characterization of benign cells. ${ }^{8}$ Another issue is the heterogeneity of biomarkers expressed in CTCs of various cancer types, ${ }^{9}$ such as epithelial markers (EpCAM, ${ }^{10}$ epithelial glycoprotein 2 (EGP-2), ${ }^{11}$ and cytokeratins ${ }^{12}$ ), tumor-related markers (carcinoembryonic antigen (CEA) ${ }^{13}$ alpha-fetoprotein (AFP), ${ }^{14}$ and squamous-cell carcinoma antigen (SCCA) ${ }^{15}$ ), mRNAs (human telomerase reverse transcriptase (hTERT) ${ }^{16}$ and telomerase ${ }^{17}$ ) and organ-specific markers (HER2-neu, ${ }^{18}$ prostate-specific antigen (PSA), ${ }^{19}$ mammaglobin, ${ }^{20}$ etc.). Apparently, it is very challenging to rely on a single type of biomarker for the highly specific isolation of extremely rare CTCs in complex clinical samples from patients with different cancer types.

Microfluidics, capable of precise microscale manipulation, has led to the development of a variety of cell sorting techniques, which generally can be classified as active and passive methods depending on the means of force field generation. Active sorting methods usually introduce external acoustic, ${ }^{21-23}$ electric $^{24-26}$ and magnetic ${ }^{27,28}$ fields to selectively manipulate different cell populations. Their high accuracy and tunable cell sorting capability, however, result in low throughput sample processing. Passive methods are typically size-based label-free 
sorting techniques, including microfiltration, ${ }^{29}$ deterministic lateral-displacement (DLD) ${ }^{30}$ and inertial focusing. ${ }^{31-33}$ These passive sorting methods hold great advantages of high throughput sample handling, cost-effective device fabrication, simple experimental setup and low energy consumption. To address the aforementioned problems, researchers have utilized inertial focusing to collect rare target cells from complex samples with high throughput. Fei et al. ${ }^{34}$ utilized nonNewtonian fluids within a straight channel to achieve the collection of MCF-7 cancer cells with an enrichment ratio of around 750-fold. Hou et al. ${ }^{12}$ achieved around 10\% CTC purity in a spiral channel with a rectangular cross-section. Warkiani et $a .^{35}$ attained ultra-high throughput with purity of CTCs of $0.6-25 \%$ in a slanted spiral chip (with a trapezoid cross-section) by vertically parallelizing the spiral chip. However, these labelfree cell sorting techniques mainly rely on the size difference between CTCs and blood cells, thus lacking sufficient sorting specificity.

Therefore, a hybrid cell sorting system combining the merits of active and passive methods shows great potential to implement high throughput, high accuracy cell sorting of complex biological samples to address real-world problems. In recent years, there has been an increasing interest in exploring the use of acoustic waves for active particle and cell sorting according to their density, size and compressibility. ${ }^{36-41}$ Compared with dielectrophoresis and magnetophoresis, acoustophoresis-based active sorting exerts no or minimal damage on biological cells ${ }^{42}$ and works in all aqueous solutions without any special requirements. Dielectrophoresis with strong electric fields and generated heat may damage cells and only works in aqueous solutions with certain electrical properties, in particular, electrical conductivity. Magnetophoresis involves magnetic beads and requires extra incubation time and elution steps to remove magnetic beads from isolated cells. In particular, we have demonstrated the application of a highly focused acoustic beam $(\sim 25 \mu \mathrm{m} \text { wide })^{43}$ for high accuracy single cell level sorting at $\sim \mathrm{kHz}$ rates ${ }^{44}$ It is ideal to process pre-enriched cell samples using our acoustic single cell sorting device to significantly shorten the whole sorting process. In this study, we have developed the first hybrid microfluidic cell sorting technique combining passive inertial cell enrichment ${ }^{45}$ and active acoustic single cell sorting, which provides a promising solution for high throughput and high accuracy isolation of rare cell populations needed for practical biomedical applications. In order to demonstrate the capability of this hybrid cell sorting technique and its potential in clinical applications, we applied this technique to isolate lowabundance breast cancer cells spiked in whole blood samples mimicking clinical samples from cancer patients. We have successfully achieved at least 2500-fold enrichment of target cancer cells and maintained high cell viability to proliferate after the whole hybrid sorting process. The high cell viability enables versatile post single cell analysis, for example, single cell genome sequencing and in vitro culture of rare cells, which is greatly beneficial for cancer study, diagnosis and treatment.

\section{Concept and operating principle}

\section{Principle of size-based passive inertial sorting}

When a particle flows in a straight channel in an intermediate Reynolds number regime $(\sim 100>\operatorname{Re}>\sim 1)$, it is subjected to a nontrivial lateral inertial lift force $\left(F_{\mathrm{L}}\right)$ consisting of a shear gradient-induced lift force and wall-induced lift force ${ }^{46}$ and obeying the relationship expressed as follows: ${ }^{47,48}$

Central region:

$$
F_{\mathrm{L}}=\frac{f_{\mathrm{L}} \rho_{\mathrm{f}} U^{2} d^{4}}{H^{2}}
$$

Near-wall region:

$$
F_{\mathrm{L}}=\frac{f_{\mathrm{L}} \rho_{\mathrm{f}} U^{2} d^{6}}{H^{4}}
$$

In the above, $f_{\mathrm{L}}$ is the lift coefficient, and $\rho_{\mathrm{f}}, U$ and $d$ refer to the fluid density, fluid average velocity and particle diameter, respectively. $H$ is defined as the hydraulic diameter and calculated in a rectangular channel as $2 w h /(w+h)$, where $w$ and $h$ refer to the channel width and height of the cross-section, respectively.

For microfluidic channels with a curvature or an expansionconstriction structure, a secondary flow perpendicular to the main flow direction, called Dean flow, will occur in the form of two counter-rotating Dean vortices, resulting from the inertia mismatch of the continuous flow in the central and near-wall regions. Accordingly, the Dean flow exerts an additional Dean drag force $\left(F_{\mathrm{D}}\right)$ on the flow-through particles perpendicular to the main flow, and it is expressed as

$$
F_{\mathrm{D}}=3 \pi \mu d U_{\mathrm{D}} \sim \frac{\rho_{\mathrm{f}} U^{2} d H^{2}}{R},
$$

where $\mu$ is the fluid viscosity, $U_{\mathrm{D}}$ is the magnitude of the Dean flow and $R$ is the radius of the curvature. Notably, the Dean drag force scales distinctively with the particle diameter as compared with the inertial lift force. As a result, the balance between the two forces gives rise to size-dependent differential particle focusing, which produces the differential equilibrium positions of varying particle sizes for size-based particle sorting in a continuous flow. ${ }^{48}$

Fig. 1a shows the schematic of the size-based inertial cell sorting, particularly in a reverse wavy channel unit near the trifurcated output, which is the optimized microchannel design for enriching cancer cells from blood cells. ${ }^{45}$ Fluorescently labeled cancer cells are spiked into whole blood samples. The inertial sorting device has two inlets, one for the prepared cell sample and another for sheath flow, in which the sheath flow occupies the central region to initially confine cells to the wall regions. The main channel has a width of $125 \mu \mathrm{m}$ and a height of $40 \mu \mathrm{m}$. The width of the trifurcated outlet branch is $80 \mu \mathrm{m}, 45 \mu \mathrm{m}$ and $80 \mu \mathrm{m}$. Larger cancer cells are expected to be dominated by the inertial lift force and focused along the centerline of the channel, while smaller blood cells are dominated by the Dean drag force and focused in the regions near the two side channel walls. As a result, the middle outlet of the trifurcation can collect the pre- 

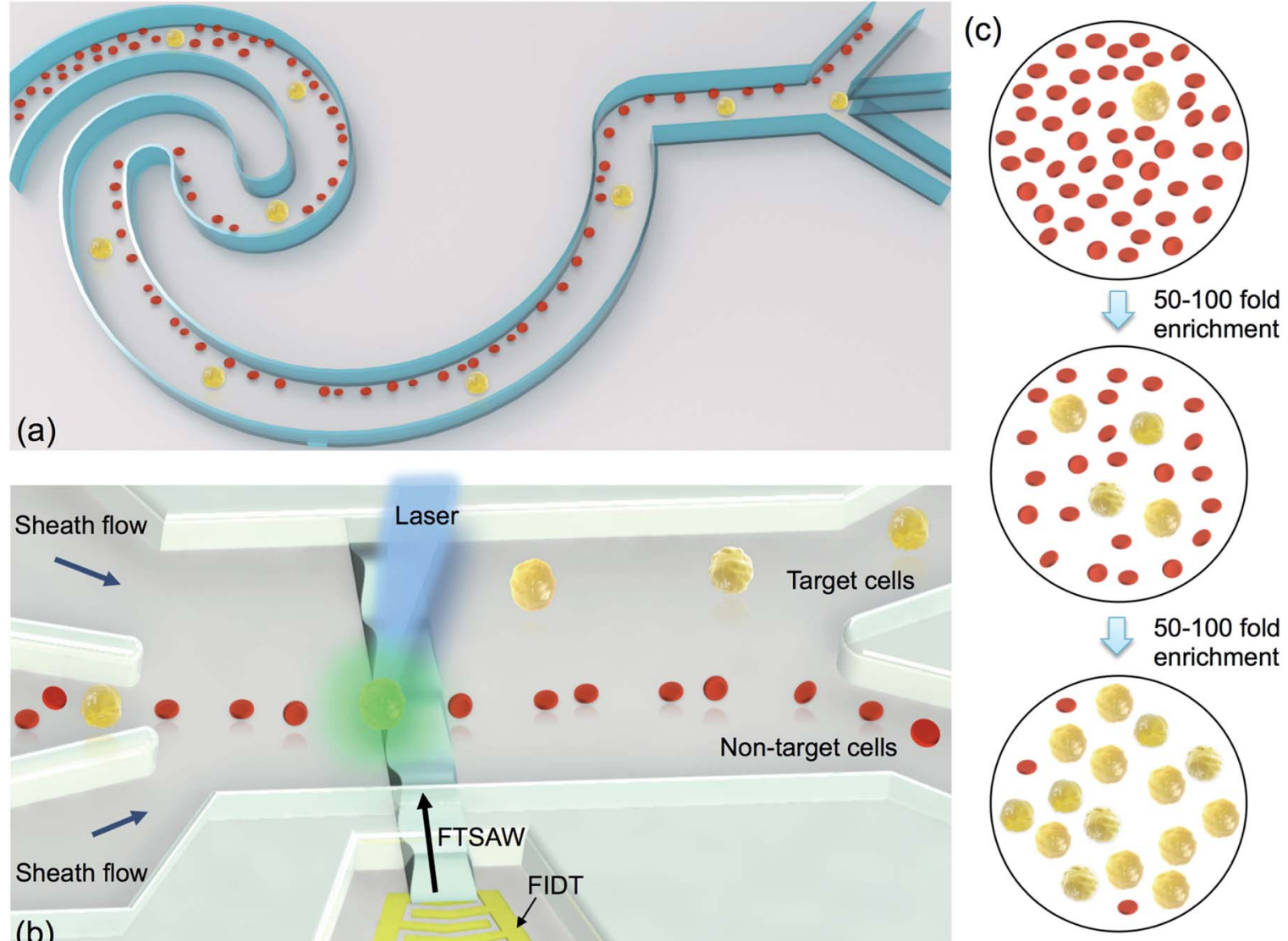

(b)

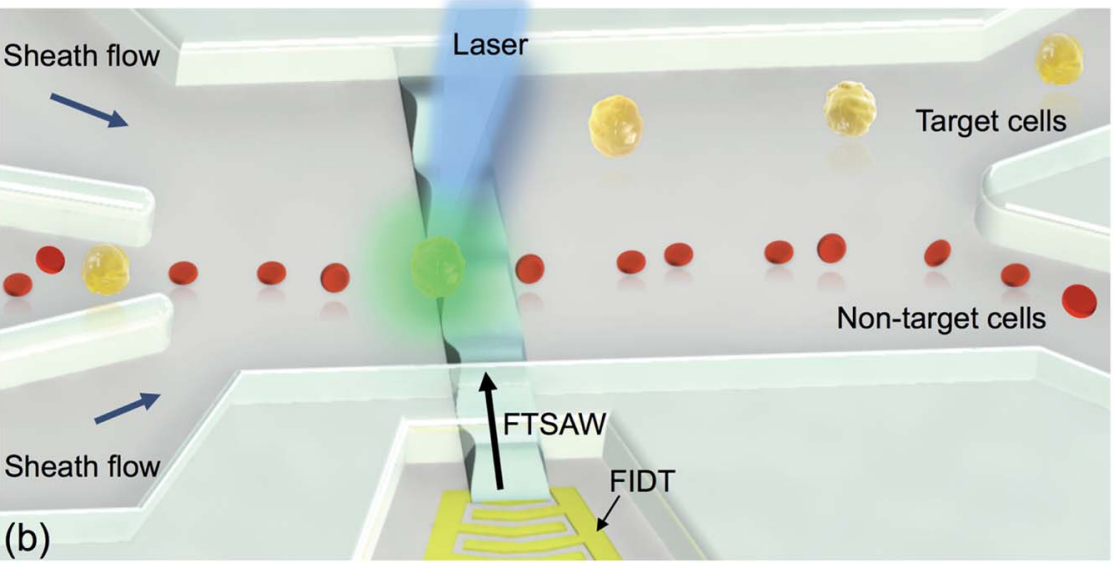

Fig. 1 Hybrid sorting method combining inertial focusing and acoustic manipulation for rare cell isolation. (a) Schematic of the size-based inertial cell sorting device (one reverse wavy channel unit near the trifurcated section). Cancer cells and blood cells are expected to have differential focusing positions because of the size difference. (b) Schematic of the fluorescence-activated acoustic sorting at the single cell level. Sandwiched by two asymmetric sheath flows, the cell sample suspension flows through the fluorescence interrogation region where the fluorescent label on target cancer cells is excited. Upon the detection of single cell fluorescence emission, the focused interdigital transducer (FIDT) generates a focused acoustic wave beam to laterally transport the detected target cell toward the target outlet, while the non-target cells follow the original streamline to the waste outlet. (c) Schematic of the cell content after each enrichment cycle. Size-based inertial sorting and fluorescence activated acoustic sorting can both enrich the target cells 50-100 fold, resulting in an overall 2500-10 000 fold enrichment that is typically needed for rare cell isolation.

enriched cancer cells based on the size difference. It is expected that the inertial cell sorting can enrich the target cells 50- to 100fold solely based on the size difference.

\section{Principle of fluorescence-activated acoustic sorting}

Fig. $1 \mathrm{~b}$ shows the schematic of the fluorescence activated acoustic sorting of a single target cell. The pre-enriched cell sample by inertial sorting is continuously infused into the acoustic cell sorting device, including a microfluidic channel and a focused interdigital transducer (FIDT). Two sheath flows hydrodynamically confine all the cells into a single file so that they pass through a laser spot for fluorescence interrogation one-by-one. Once the fluorescent label on a target cell is detected using a photomultiplier tube (PMT), it applies a pulsed AC signal to activate the FIDT for producing a highly focused acoustic beam. The pulsed acoustic wave propagates along the substrate surface and radiates into the fluid at the substratefluid interface. The absorption of acoustic energy generates a pressure gradient along the wave propagation, and thus produces an acoustic streaming flow (ASF). In our most recent study, we successfully applied this localized ASF produced by a highly focused acoustic beam to isolate single biological cells. ${ }^{44}$ Different from the size-based inertial sorting, the proposed acoustic sorting can isolate target cells at the single cell level based on their specific surface biomarkers. It is expected that the fluorescence-activated acoustic sorting can enrich the target cells by another 50- to 100-fold. Thus, the hybrid sorting process is capable of enriching target cells by 2500-10 000-fold, providing a new solution of rare cell isolation.

\section{Materials and methods}

\section{Device fabrication}

The inertial cell sorting devices were fabricated using a standard polydimethylsiloxane (PDMS) soft-lithography protocol. Briefly, the master mold for PDMS casting was made with SU-8 (SU-8 2025, MicroChem, Newton, MA, USA) on a silicon wafer using photolithography. The PDMS channel was fabricated by casting 
a liquid PDMS mixture onto the pre-fabricated master mold to replicate the channel feature on the mold. Once the PDMS layer with the channel features was fully cured by heat, it was demolded and treated with air plasma (Harrick Plasma PDC-32G, Ithaca, NY, USA), which aimed at generating a hydroxyl functional group on the surface. A separate glass slide was bonded onto the PDMS channel layer to form a closed microfluidic device.

The acoustic cell sorting device was fabricated via plasma bonding another PDMS channel layer onto a focused interdigital transducer (FIDT). The FIDT is a $128^{\circ}$ rotated Y-cut Xpropagation lithium niobate $\left(\mathrm{LiNbO}_{3}\right)$ substrate patterned with 36 pairs of concentric arcuate electrodes. These electrodes have a uniform spacing and width of $7.5 \mu \mathrm{m}$ and an aperture of $160 \mu \mathrm{m}$ at the proximal end, subtending an angle of $26^{\circ}$ to the distal end. They were fabricated using electron beam evaporation by depositing a $\mathrm{Cr} / \mathrm{Al}$ layer $(7 \mathrm{~nm} / 200 \mathrm{~nm})$ on the $\mathrm{LiNbO}_{3}$ substrate, and then another $300 \mathrm{~nm}$ thick $\mathrm{SiO}_{2}$ layer on the top to prevent corrosion and promote bonding with the PDMS layer. After a 150 seconds air plasma treatment, the PDMS channel layer and the FIDT were aligned and permanently bonded together to form the acoustic cell sorting device.

\section{Cell culture}

The MCF-7 breast cancer cell line was purchased from American Type Culture Collection (ATCC cat. no. HB-72), cultured with Dulbecco's Modification of Eagle's Medium (DMEM, Thermo Fisher Scientific, USA) and supplemented with 10\% fetal bovine serum (FBS, Thermo Fisher Scientific, USA) to provide growth factors and antibiotics, including penicillin and streptomycin (Thermo Fisher Scientific, USA) to prevent growth of bacteria. Cells were sub-cultured every 2 to 3 days when the monolayer reached $80-90 \%$ confluence and maintained at $37{ }^{\circ} \mathrm{C} 5 \%(\mathrm{v} / \mathrm{v})$ $\mathrm{CO}_{2}$ in a humidified incubator. During the subculture procedures, cells were trypsinized with a $0.25 \%$ trypsin-EDTA solution (Thermo Fisher Scientific, USA).

\section{Sample preparation}

Fluorescent polystyrene microspheres $(15 \mu \mathrm{m}, 10 \mu \mathrm{m}, 7 \mu \mathrm{m}$ and $3 \mu \mathrm{m}$ ) were purchased without any further modification (Magsphere, USA). To avoid particle agglomeration and adhesion onto the microchannel walls, these fluorescent polystyrene particles were diluted with deionized (DI) water containing 0.6\% Pluronic F127 (Sigma-Aldrich, USA). The typical particle concentration used in these experiments was around $6 \times 10^{6}$ particles per ml. Cancer cells (MCF-7) were stained with a SYTO 9 fluorescent dye (Thermo Fisher Scientific, USA) and spiked in diluted whole blood (final concentration around $5 \times 10^{8}$ cells per $\mathrm{ml}$ ) with initial purity of around $0.01 \%$. This cell mixture was used to mimic clinical samples aiming to demonstrate potential applications of combining inertial sorting for sizebased CTC enrichment and acoustic sorting for fluorescenceactivated CTC isolation at the single cell level.

\section{Cell viability test}

The sorted cells, collected from the target outlet of the inertial sorting device and the acoustic sorting device, were further enriched by centrifugation to reach a comparable concentration as that of the input pre-mixed sample. Subsequently, the three samples from the inlet and target outlets were, respectively, mixed with a $0.4 \%$ trypan blue stain (Thermo Fisher Scientific, USA) in a volume ratio of 9 to 1 . Each of the three mixtures was then separately injected into a cell count board to take microscopic photographs within $3 \mathrm{~min}$. The cell viability was evaluated by the ratio of the unstained cell number to the total cell number.

\section{Experimental setup}

A new cell sorting device was used in each repeated experiment to avoid cross contamination. In the inertial sorting experiments, the as-prepared cell sample and the sheath flow (Dulbecco's phosphate-buffered saline, DPBS, Thermo Fisher Scientific, USA) were continuously infused into the inertial sorting device using two separate syringe pumps. The trajectories of fluorescent microparticles were recorded using a CCD camera on an inverted microscope (Olympus, CKX53, Japan) to capture their inertial focusing behavior. For performing cancer cell enrichment, the cell sample collected from the middle outlet of the inertial sorting device was used in acoustic sorting for refined single cell isolation.

The fluorescence-activated acoustic sorting system consists of three main parts, as shown in Fig. 2: (i) fluorescence excitation and detection subsystem, (ii) acoustic cell sorting subsystem and (iii) observation stage. The fluorescence excitation and detection subsystem excites the fluorescent label specifically bound to the target cells and converts the fluorescence emission into an electrical signal to trigger the acoustic cell sorting subsystem. Briefly, a blue laser beam was emitted from a $473 \mathrm{~nm}$ wavelength laser source, reflected by the dichroic beam splitter (D2), and then focused by a convex lens (L1) into a tiny laser spot $(\sim 70 \mu \mathrm{m}$ in diameter $)$ as the fluorescence

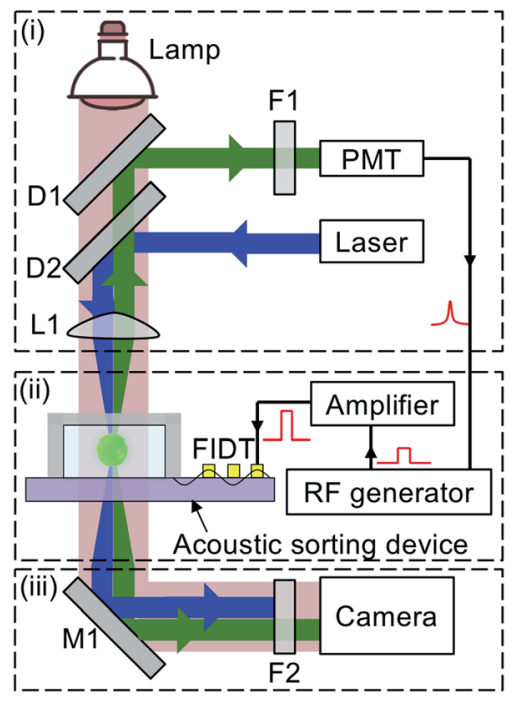

Fig. 2 Schematic of the fluorescence-activated acoustic cell sorting system, which is composed of three main parts: (i) fluorescence excitation and detection subsystem, (ii) acoustic cell sorting subsystem and (iii) observation stage. 
interrogation region in the microfluidic channel. Once a fluorescently labeled target cell enters the laser spot, it is excited to emit light with a longer wavelength than that of the excitation light. The emission transmits through L1 and D2, and then gets reflected by another dichroic beam splitter D1 and filtered by a bandpass filter F1 into the PMT, which converts the fluorescence signal into a high-level electrical pulse signal. Upon each high-level signal output from the PMT, the RF generator produces a pulsed $1 \mathrm{~ms}$ AC signal with a delay of $0.1 \mu \mathrm{s}$ that is amplified by a power amplifier, and then applied on the FIDT to generate a highly focused SAW beam for single cell level sorting. The sorting process was illuminated by a lamp and captured by a high-speed camera (Photron Inc., San Diego, CA, USA).

\section{Results and discussion}

\section{Inertial sorting for size-based CTC enrichment}

Fig. 3 shows the steady inertial focusing behavior of differently sized microspheres in our inertial sorting device and their corresponding collection at the trifurcated output. CTCs typically have sizes between 15 and $25 \mu \mathrm{m}$; therefore, we used a $15 \mu \mathrm{m}$ fluorescent microsphere to represent the minimum size of CTCs, which was focused as one single fluorescent streak along the centerline of the channel (Fig. 3a). A $10 \mu \mathrm{m}$ fluorescent microsphere (Fig. 3b) was used to mimic white blood cells $(7-30 \mu \mathrm{m})$, among which at least $93 \%$ were smaller than $15 \mu \mathrm{m} .{ }^{49}$ A $7 \mu \mathrm{m}$ fluorescent microsphere (Fig. 3c) was used to mimic red blood cells $(6-8 \mu \mathrm{m})$, and both 7 and $10 \mu \mathrm{m}$ microspheres exhibited one single streak focusing. Notably, due to different scaling with the particle diameter under the inertial lift force and Dean drag force, the $15 \mu \mathrm{m}$ microspheres were largely dominated by the inertial lift force such that they stayed nearly along the centerline and assembled in the middle outlet. Although the inertial lift force also dominated the Dean drag force for the $7 \mu \mathrm{m}$ and $10 \mu \mathrm{m}$ microspheres, the increased Dean drag force shifted the equilibrium focusing position towards the upper channel wall. As a result, both $7 \mu \mathrm{m}$ and $10 \mu \mathrm{m}$ microspheres were collected at the upper outlet. A $3 \mu \mathrm{m}$ fluorescent microsphere (Fig. 3d) was mimicking platelets $(2-4 \mu \mathrm{m})$, and its inertial focusing behavior was found to be dominated by the Dean drag force. Because the repeated reverse wavy channel units periodically changed the Dean flow direction, the Dean drag force tended to move particles towards the channel walls, and thus formed two streaks near the two sidewalls. A more detailed analysis of size-dependent inertial focusing in our novel inertial sorting device was discussed in our previous study. ${ }^{45}$

To further demonstrate the clinical potential of this novel inertial sorting device for rare cell isolation, fluorescently labeled breast cancer cells (MCF-7, diameter around 19-24 $\mu \mathrm{m}$ ) spiked in diluted whole blood samples with purity of $\sim 0.02 \%$ were used as the input cell samples. Due to the possible cell-cell interaction at a $10 \mathrm{X}$ dilution of a human whole blood sample, a sheath flow was adopted to minimize the RBC contamination of the target cell collection, in which the sheath flow confined all the cells to the sidewalls when entering the main channel. The flow rates of the cell sample and sheath flow were 80

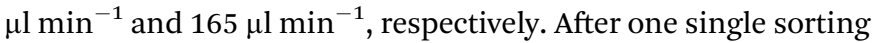
process, the sample collected from the middle outlet contained the enriched target MCF-7 cells. The originally prepared cell samples and sorted target samples were evaluated by analyzing the randomly captured microscopic cell images using a published cell statistic code. ${ }^{50}$ It was found that the size-based inertial sorting can enrich cancer cells by $\sim 61$-fold with their purity increased from $0.02 \%$ to $1.23 \%$.

\section{Fluorescence-activated acoustic sorting at single cell level}

Next, we demonstrated our capability of isolating target cells at the single cell level based on fluorescence-activated acoustic sorting. Fig. 4 shows the sorting process of a single MCF-7 cancer cell from the human blood cells. The flow rate of the upper sheath flow, the middle sample flow and the lower sheath flow

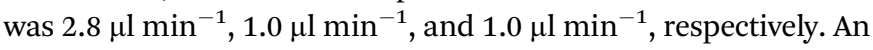
AC signal with the power of $45 \mathrm{~mW}$ and duration of $1 \mathrm{~ms}$ was applied to the FIDT to produce a pulsed SAW beam with a width of $\sim 50 \mu \mathrm{m}$. The laser spot for fluorescence excitation was aligned with a highly focused acoustic beam. Upon the detection of fluorescence emission from a single target cell, the pulsed SAW beam was instantaneously generated to laterally translate the

\begin{tabular}{c|c}
\hline Cell Type & Size $(\mu \mathrm{m})$ \\
\hline Cancer cells & $15-25$ \\
\hline 0 White blood cells & $7-30$ \\
\hline$\quad$ Red blood cells & $6-8$ \\
\hline$\quad$ platelets & $2-4$ \\
\hline
\end{tabular}
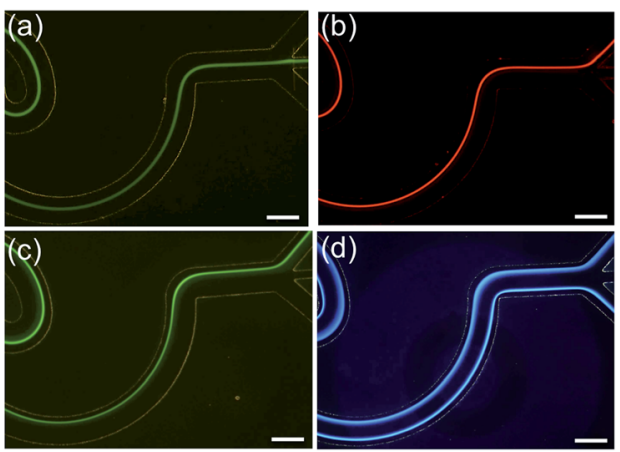

Fig. 3 Inertial focusing behavior of differently sized microspheres in the inertial sorting device. (a) $15 \mu \mathrm{m}$ fluorescent microsphere is used to represent the minimum size of cancer cells. (b) $10 \mu \mathrm{m}$ fluorescent microsphere is used to mimic white blood cells. (c) $7 \mu \mathrm{m}$ fluorescent microsphere is used to mimic red blood cells. (d) $3 \mu \mathrm{m}$ fluorescent microsphere is used to mimic platelets. Scale bar is $125 \mu \mathrm{m}$. 


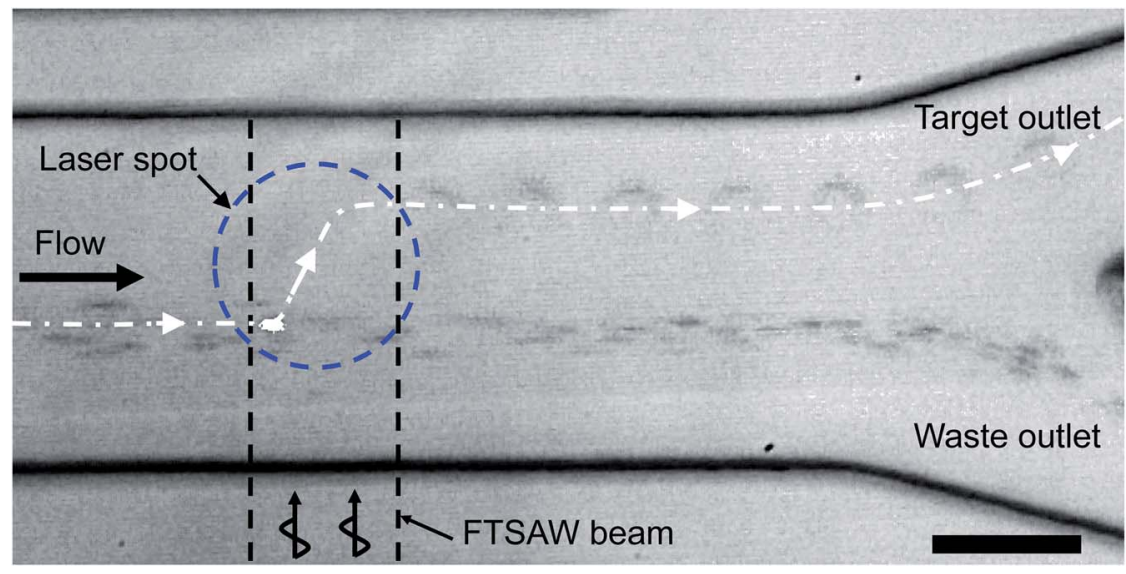

Fig. 4 Time-lapsed microscopic image showing the process of sorting one single fluorescently labeled MCF-7 cancer cell from blood cells to the upper target outlet. Unlabeled non-target blood cells flow into the lower waste outlet. The image was obtained by overlapping 12 frames recorded every $2 \mathrm{~ms}$. The scale bar is $50 \mu \mathrm{m}$.

detected single cell to the upper target outlet. In contrast, the blood cells without a fluorescent label followed the original streamline to the lower waste outlet. A series of fluorescenceactivated single cell sorting processes can be visualized in the ESI Video S1. $\uparrow$ The purity of the fluorescently labeled cancer cells in the input sample and collected target sample was respectively characterized as $0.75 \%$ and $41.68 \%$ based on the aforementioned imaging-based single cell analysis code..$^{50}$

\section{Hybrid sorting for rare cell isolation}

Inertial sorting is a size-based cell enrichment technique and is thus ideal for pre-enrichment to deplete a majority of non-target cells. Although CTCs are larger than the majority of blood cells, they still have a considerable size overlap with white blood cells. In addition, inertial sorting typically processes highly concentrated cell suspensions to fulfill the throughput requirement, and cell-cell interactions can contaminate the target outlet. Fluorescence-activated acoustic sorting can isolate single cells based on the fluorescent labels specifically bound to the target cells. In particular, the ability to produce a highly focused acoustic beam enables high accuracy isolation at the single cell level. In the following demonstration of hybrid sorting, we first applied inertial sorting for high throughput size-based enrichment, and then used fluorescence-activated acoustic sorting for high accuracy single cell level isolation.

Fluorescently labeled breast cancer cells spiked in diluted $(10 \times)$ whole blood samples with the purity of $\sim 0.01 \%$ were used to mimic a clinical sample containing rare target cells. The concentration of the target cells could be further decreased, but we could not accurately quantify the initial cell concentration using our imaging-based single cell analysis code. Fig. 5a shows one example of the microscopy images of the simulated input sample containing rare cancer cells. The input sample first went through our inertial sorting device, and we collected the sample from the middle outlet (i.e., cells larger than $15 \mu \mathrm{m}$, as shown in Fig. 5b). The pre-enriched cell suspension was then reinjected and went through our acoustic sorting device. Fig. $5 \mathrm{c}$ shows one of the microscopic images of the output sample after the entire hybrid sorting process. The hybrid sorting experiments were repeated three times under the same conditions, and twenty microscopy images of cell samples were captured after each sorting experiment for the cell content analysis. Fig. 5d shows the detailed experimental results before and after each sorting process. Fig. 5a-c are randomly chosen from a series of captured microscopy images for data analysis and do not represent the exact value of cancer cell purity listed in Fig. 5d.

Most of the reported hybrid sorting methods do not provide the target cell enrichment ratio, and thus only present the depletion rate of white blood cells. For acoustophoresis-based hybrid methods, Mutafopulos et al. ${ }^{51}$ adopted inertial focusing with a spiral channel to pre-align the cells as a single line, and then used acoustic fluorescence-activated cell sorting to enhance the purity of the spiked cancer cells by $\sim 20$-fold. For magnetophoresis-based hybrid methods, Ozkumur et al. ${ }^{\mathbf{2}}$ developed a hybrid sorting chip called CTC-iChip, which processed whole blood spiked with cancer cells through DLD-based cell sorting first, followed by inertial focusing and immunomagnetic sorting of cancer cells from white blood cells, eventually achieving roughly 1000-fold enrichment of rare CTCs. Masahiro et al. ${ }^{53}$ used hydrodynamic filtration combined with immunomagnetic beads to demonstrate the size-based and surface marker-based sorting of a mixture of human lymphocyte cells (JM cells) and HeLa cells, in which the purity of the immunomagnetic labeled lymphocyte cells was increased from $41 \%$ to $92 \%$ (around $\sim 2.2$-fold). Sajay et al. ${ }^{54}$ conducted immunomagnetic negative selection to deplete more than $90 \%$ of white blood cells from whole blood spiked with MCF-7 breast cancer cells and then processed the sample with a microfabricated filter membrane to collect the target MCF-7 cells. Giudice et al. ${ }^{55}$ demonstrated deterministic particle separation by feeding magnetic particles into a viscoelasticity medium to achieve viscoelastic focusing first, followed by continuous flow through a magnetic field for an efficient magnetic particle separation $(\sim 96 \%)$. For the dielectrophoresis-based hybrid methods, Chang et $a l .{ }^{56}$ combined DLD and the electrical 


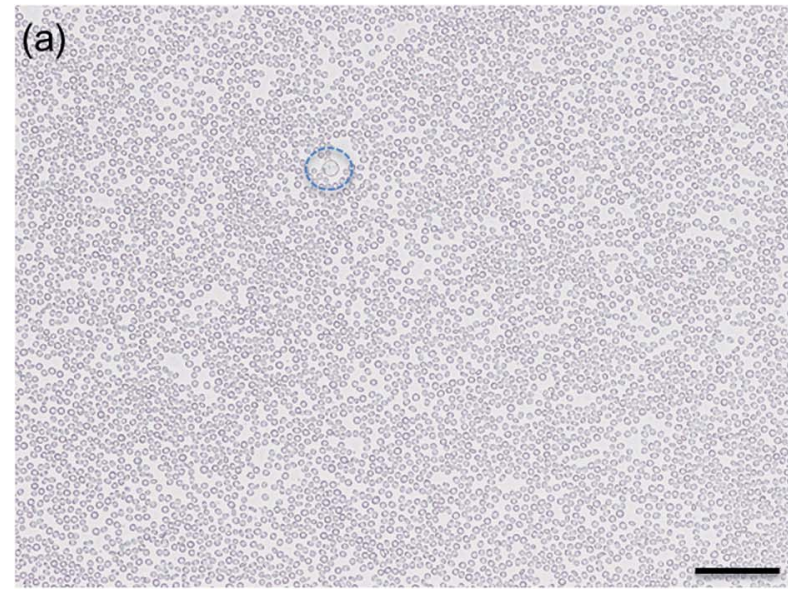

(c)
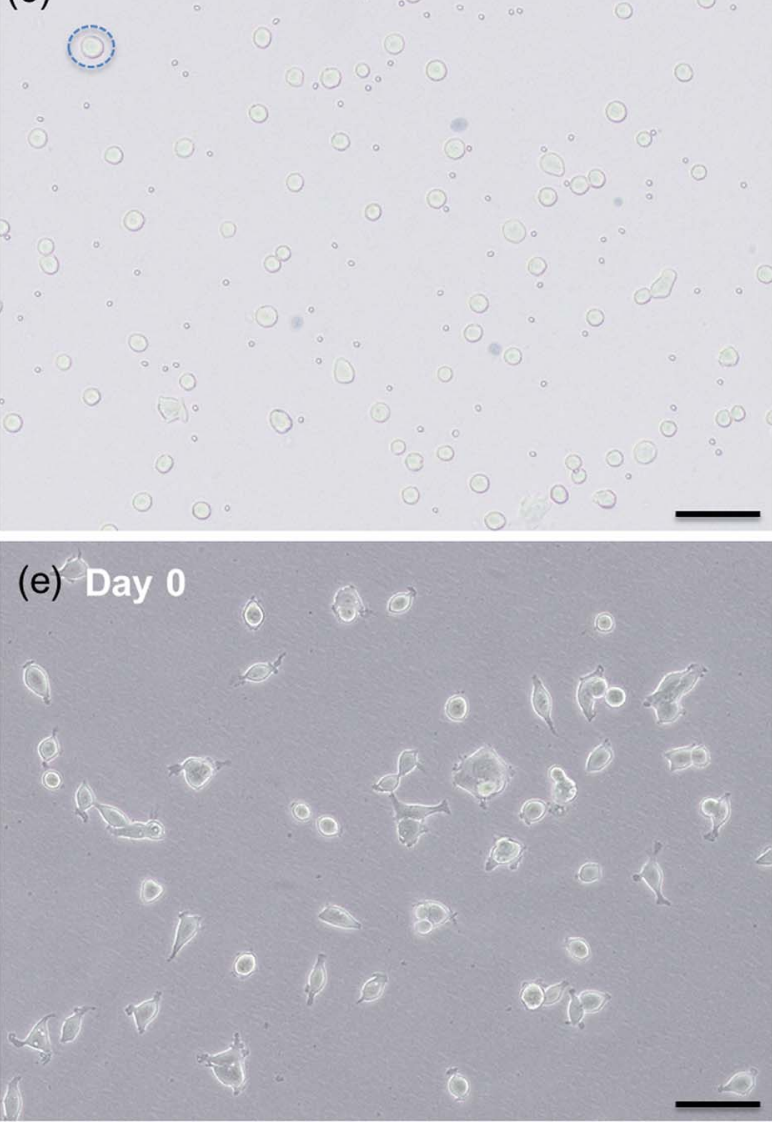

(b)

(d)

\begin{tabular}{ccccc} 
Experiments & 1 & 2 & 3 & mean \\
\hline Purity 1 (\%) & 0.011 & 0.016 & 0.015 & $0.014 \pm 0.003$ \\
\hline Purity 2 (\%) & 0.65 & 0.68 & 0.64 & $0.66 \pm 0.02$ \\
\hline Purity 3 (\%) & 41.67 & 42.26 & 36.51 & $40 \pm 3$ \\
\hline Viability 1 (\%) & 95.8 & 94.2 & 92.3 & $94 \pm 2$ \\
\hline Viability 2 (\%) & 90.7 & 90.1 & 89.3 & $90 \pm 1$ \\
\hline Viability 3 (\%) & 92.3 & 91.1 & 89.8 & $91 \pm 1$ \\
\hline Enrichment ratio & 3788 & 2642 & 2434 & $3000 \pm 700$ \\
\hline
\end{tabular}

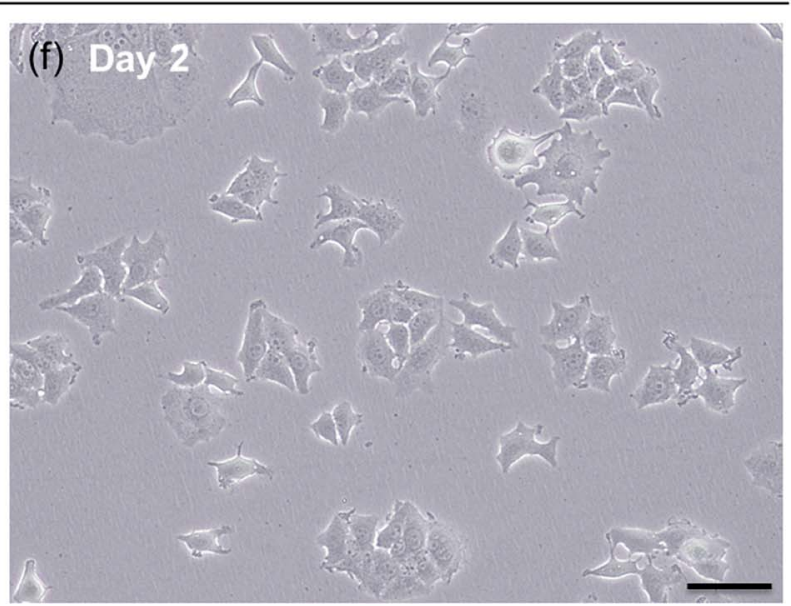

Fig. 5 Representative microscopic photographs of cell samples collected from (a) input inlet of inertial sorting device, (b) target outlet of inertial sorting device, and (c) target outlet of acoustic sorting device. A representative cancer cell in each sample is indicated by a blue dashed circle. Scale bar is $100 \mu \mathrm{m}$. (d) Experiments 1-3 refer to the three repeated experiments under the same sorting conditions. Purity 1 and viability 1 refer to the purity and viability of cancer cells from sample (a). Purity 2 and viability 2 refer to the purity and viability of cancer cells from sample (b). Purity 3 and viability 3 refer to the purity and viability of cancer cells from sample (c). (e) and (f) show proliferation of cancer cells collected from the final sorting output.

method, replacing the physical pillar array with dielectrophoretic virtual pillar array, and achieved 99\% separation of red blood cells from white blood cells. Moon et al. ${ }^{57}$ separated MCF7 spiked in whole blood samples, first with multi-orifice flow fractionation, followed by dielectrophoresis, achieving a 162fold enrichment of MCF-7 cells.
By contrast, using our hybrid sorting technique, we reliably achieved at least 2500 -fold cancer cell enrichment with purity increased from $\sim 0.01 \%$ to $\sim 40 \%$. In addition, it is unlike the existing hybrid dielectrophoresis method that may damage cells due to the heat generated by strong electrical fields and only works for aqueous solutions with certain electrical conductivity. It is also different from the hybrid magnetophoresis method 
that involves magnetic bead intervention and requires extra incubation time and elution steps to remove magnetic beads from isolated cells. Our developed hybrid sorting method can maintain cell viability as high as $91 \pm 1 \%$ (viability before sorting is $94 \pm 2 \%$ ) and works for all aqueous solutions without any special requirements. After hybrid sorting, the collected cells were cultured (without the trypan blue test) and showed great proliferation (Fig. 5e and f), which enables versatile analysis and manipulation of isolated rare cells.

\section{Conclusion}

In this study, for the first time, we present a hybrid microfluidic cell sorting method combining passive size-based inertial sorting and active surface biomarker-based acoustic sorting. Here, we adopted a novel geometric channel design with a reverse wavy channel to implement differential cell focusing for high throughput size-based enrichment. The pre-enriched rare cells were specifically labeled with fluorescent dyes and passed through an acoustic cell sorting device, in which a highly focused acoustic beam could be activated upon the detection of fluorescent labels to accurately isolate target cells at the single cell level. To demonstrate the capability of this hybrid sorting technique for rare cell isolation, we used whole blood samples spiked with MCF-7 breast cancer cells at the concentration of around $0.01 \%$ to mimic clinical samples from cancer patients. Several hybrid sorting experiments showed that at least 2500fold cancer cell enrichment could be reliably achieved, and the collected target cells could maintain proliferation after the entire hybrid sorting process. This hybrid sorting technique possesses the advantages of high throughput size-based enrichment and high accuracy surface biomarker-based single cell isolation, thus providing a new means of reliable rare cell isolation in cancer study, diagnosis and treatment.

\section{Conflicts of interest}

There are no conflicts to declare.

\section{Acknowledgements}

This study was supported by Singapore Ministry of Education Academic Research Fund Tier 2 (T2MOE1603) awarded to YA.

\section{References}

1 A. Jemal, F. Bray, M. M. Center, J. Ferlay, E. Ward and D. Forman, Global cancer statistics, Ca-Cancer J. Clin., 2011, 61(2), 69-90.

2 T. R. Ashworth, A case of cancer in which cell similar to those in the tumours were seen in the blood after death, Med. J. Aust., 1869, 14, 146-149.

3 E. Racila, D. Euhus, A. J. Weiss, C. Rao, J. McConnell, L. W. Terstappen and J. W. Uhr, Detection and characterization of carcinoma cells in the blood, Proc. Natl. Acad. Sci. U. S. A., 1998, 95(8), 4589-4594.
4 G. P. Gupta and J. Massagué, Cancer Metastasis: Building a Framework, Cell, 2006, 127, 679-695.

5 J. P. Thiery and C. T. Lim, Tumor dissemination: an EMT affair, Cancer Cell, 2013, 23, 272-273.

6 A. Bonnomet, A. Brysse, A. Tachsidis, M. Waltham, E. W. Thompson, M. Polette and C. Gilles, Epithelial-tomesenchymal transitions and circulating tumor cells, $J$. Mammary Gland Biol. Neoplasia, 2010, 15(2), 261-273.

7 J. M. Pawelek and A. K. Chakraborty, Fusion of tumour cells with bone marrow-derived cells: a unifying explanation for metastasis, Nat. Rev. Cancer, 2008, 8(5), 377-386.

8 A. M. Sieuwerts, J. Kraan, J. Bolt, P. van der Spoel, F. Elstrodt, M. Schutte, J. W. Martens, J. W. Gratama, S. Sleijfer and J. A. Foekens, Anti-epithelial cell adhesion molecule antibodies and the detection of circulating normal-like breast tumor cells, J. Natl. Cancer Inst., 2009, 101(1), 61-66.

9 A. L. Allan and M. Keeney, Circulating tumor cell analysis: technical and statistical considerations for application to the clinic, J. Oncol., 2010, 2010, 426218.

10 F. Farace, C. Massard, N. Vimond, F. Drusch, N. Jacques, F. Billiot, A. Laplanche, A. Chauchereau, L. Lacroix, D. Planchard, S. Le Moulec, F. Andre, K. Fizazi, J. C. Soria and P. Vielh, A direct comparison of CellSearch and ISET for circulating tumour-cell detection in patients with metastatic carcinomas, Br. J. Cancer, 2011, 105(6), 847-853.

11 T. J. Molloy, A. J. Bosma and L. J. van't Veer, Towards an optimized platform for the detection, enrichment, and semi-quantitation circulating tumor cells, Breast Cancer Res. Treat., 2008, 112(2), 297-307.

12 H. W. Hou, M. E. Warkiani, B. L. Khoo, Z. R. Li, R. A. Soo, D. S. Tan, W. T. Lim, J. Han, A. A. Bhagat and C. T. Lim, Isolation and retrieval of circulating tumor cells using centrifugal forces, Sci. Rep., 2013, 3, 1259.

13 C. H. Wu, S. R. Lin, F. J. Yu, D. C. Wu, Y. S. Pan, J. S. Hsieh, S. Y. Huang and J. Y. Wang, Development of a highthroughput membrane-array method for molecular diagnosis of circulating tumor cells in patients with gastric cancers, Int. J. Cancer, 2006, 119(2), 373-379.

14 A. L. Hautkappe, M. Lu, H. Mueller, A. Bex, A. Harstrick, M. Roggendorf and H. Ruebben, Detection of germ-cell tumor cells in the peripheral blood by nested reverse transcription-polymerase chain reaction for alphafetoprotein-messenger RNA and beta human chorionic gonadotropin-messenger RNA, Cancer Res., 2000, 60(12), 3170-3174.

15 J. Kaganoi, Y. Shimada, M. Kano, T. Okumura, G. Watanabe and M. Imamura, Detection of circulating oesophageal squamous cancer cells in peripheral blood and its impact on prognosis, Br. J. Surg., 2004, 91(8), 1055-1060.

16 Y. L. Wu, C. Dudognon, E. Nguyen, J. Hillion, F. Pendino, I. Tarkanyi, J. Aradi, M. Lanotte, J. H. Tong, G. Q. Chen and E. Segal-Bendirdjian, Immunodetection of human telomerase reverse-transcriptase (hTERT) re-appraised: nucleolin and telomerase cross paths, J. Cell Sci., 2006, 119(13), 2797-2806.

17 T. Xu, B. Lu, Y. C. Tai and A. Goldkorn, A cancer detection platform which measures telomerase activity from live 
circulating tumor cells captured on a microfilter, Cancer Res., 2010, 70(16), 6420-6426.

18 S. Riethdorf, V. Muller, L. Zhang, T. Rau, S. Loibl, M. Komor, M. Roller, J. Huober, T. Fehm, I. Schrader, J. Hilfrich, F. Holms, H. Tesch, H. Eidtmann, M. Untch, G. von Minckwitz and K. Pantel, Detection and HER2 expression of circulating tumor cells: prospective monitoring in breast cancer patients treated in the neoadjuvant GeparQuattro trial, Clin. Cancer Res., 2010, 16(9), 2634-2645.

19 J. S. de Bono, H. I. Scher, R. B. Montgomery, C. Parker, M. C. Miller, H. Tissing, G. V. Doyle, L. W. Terstappen, K. J. Pienta and D. Raghavan, Circulating tumor cells predict survival benefit from treatment in metastatic castration-resistant prostate cancer, Clin. Cancer Res., 2008, 14(19), 6302-6309.

20 W. H. Kruger, R. Jung, B. Detlefsen, S. Mumme, A. Badbaran, J. Brandner, H. Renges, N. Kroger and A. R. Zander, Interference of cytokeratin-20 and mammaglobin-reversetranscriptase polymerase chain assays designed for the detection of disseminated cancer cells, Med. Oncol., 2001, 18(1), 33-38.

21 F. Petersson, L. Aberg, A. M. Sward-Nilsson and T. Laurell, Free flow acoustophoresis: microfluidic-based mode of particle and cell separation, Anal. Chem., 2007, 79(14), 5117-5123.

22 H. Mulvana, S. Cochran and M. Hill, Ultrasound assisted particle and cell manipulation on-chip, Adv. Drug Delivery Rev., 2013, 65, 1600-1610.

23 D. J. Collins, Z. Ma, J. Han and Y. Ai, Continuous microvortex-based nanoparticle manipulation via focused surface acoustic waves, Lab Chip, 2016, 17(1), 91-103.

24 J. G. Kralj, M. T. Lis, M. A. Schmidt and K. F. Jensen, Continuous dielectrophoretic size-based particle sorting, Anal. Chem., 2006, 78(14), 5019-5025.

$25 \mathrm{~J}$. Voldman, Electrical forces for microscale cell manipulation, Annu. Rev. Biomed. Eng., 2006, 8, 425-454.

26 S. Park, M. Koklu and A. Beskok, Particle trapping in highconductivity media with electrothermally enhanced negative dielectrophoresis, Anal. Chem., 2009, 81(6), 23032310.

27 M. Hejazian, W. Li and N. T. Nguyen, Lab on a chip for continuous-flow magnetic cell separation, Lab Chip, 2015, 15(4), 959-970.

28 C. Murray, E. Pao, P. Tseng, S. Aftab, R. Kulkarni, M. Rettig and D. Di Carlo, Quantitative Magnetic Separation of Particles and Cells Using Gradient Magnetic Ratcheting, Small, 2016, 12(14), 1891-1899.

29 S. Choi, T. Ku, S. Song, C. Choi and J. K. Park, Hydrophoretic high-throughput selection of platelets in physiological shear-stress range, Lab Chip, 2011, 11(3), 413-418.

30 L. R. Huang, E. C. Cox, R. H. Austin and J. C. Sturm, Continuous particle separation through deterministic lateral displacement, Science, 2004, 304(5673), 987-990.

31 M. E. Warkiani, B. L. Khoo, L. Wu, A. K. Tay, A. A. Bhagat, J. Han and C. T. Lim, Ultra-fast, label-free isolation of circulating tumor cells from blood using spiral microfluidics, Nat. Protoc., 2016, 11(1), 134-148.
32 D. Di Carlo, D. Irimia, R. G. Tompkins and M. Toner, Continuous inertial focusing, ordering, and separation of particles in microchannels, Proc. Natl. Acad. Sci. U. S. A., 2007, 104(48), 18892-18897.

33 D. Di Carlo, Inertial microfluidics, Lab Chip, 2009, 9, 30383046.

34 F. Tian, L. Cai, J. Chang, S. Li, C. Liu, T. Li and J. Sun, Labelfree isolation of rare tumor cells from untreated whole blood by interfacial viscoelastic microfluidics, Lab Chip, 2018, 18(22), 3436-3445.

35 M. E. Warkiani, B. L. Khoo, D. S. Tan, A. A. Bhagat, W. T. Lim, Y. S. Yap, S. C. Lee, R. A. Soo, J. Han and C. T. Lim, An ultra-high-throughput spiral microfluidic biochip for the enrichment of circulating tumor cells, Analyst, 2014, 139(13), 3245-3255.

36 P. Augustsson, C. Magnusson, M. Nordin, H. Lilja and T. Laurell, Microfluidic, label-free enrichment of prostate cancer cells in blood based on acoustophoresis, Anal. Chem., 2012, 84(18), 7954-7962.

37 D. J. Collins, Z. Ma and Y. Ai, Highly Localized Acoustic Streaming and Size-Selective Submicrometer Particle Concentration Using High Frequency Microscale Focused Acoustic Fields, Anal. Chem., 2016, 88(10), 5513-5522.

38 Y. Chen, M. Wu, L. Ren, J. Liu, P. H. Whitley, L. Wang and T. J. Huang, High-throughput acoustic separation of platelets from whole blood, Lab Chip, 2016, 16(18), 34663472.

39 X. Ding, S. C. Lin, M. I. Lapsley, S. Li, X. Guo, C. Y. Chan, I. K. Chiang, L. Wang, J. P. McCoy and T. J. Huang, Standing surface acoustic wave (SSAW) based multichannel cell sorting, Lab Chip, 2012, 12(21), 4228-4231.

40 X. Ding, P. Li, S. C. Lin, Z. S. Stratton, N. Nama, F. Guo, D. Slotcavage, X. Mao, J. Shi, F. Costanzo and T. J. Huang, Surface acoustic wave microfluidics, Lab Chip, 2013, 13(18), 3626-3649.

41 L. Schmid, D. A. Weitz and T. Franke, Sorting drops and cells with acoustics: acoustic microfluidic fluorescence-activated cell sorter, Lab Chip, 2014, 14(19), 3710-3718.

42 M. A. Burguillos, C. Magnusson, M. Nordin, A. Lenshof, P. Augustsson, M. J. Hansson, E. Elmer, H. Lilja, P. Brundin, T. Laurell and T. Deierborg, Microchannel acoustophoresis does not impact survival or function of microglia, leukocytes or tumor cells, PLoS One, 2013, 8(5), e64233.

43 D. J. Collins, A. Neild and Y. Ai, Highly focused highfrequency travelling surface acoustic waves (SAW) for rapid single-particle sorting, Lab Chip, 2016, 16(3), 471-479.

44 Z. Ma, Y. Zhou, D. J. Collins and Y. Ai, Fluorescence activated cell sorting via a focused traveling surface acoustic beam, Lab Chip, 2017, 17(18), 3176-3185.

45 Y. Zhou, Z. Ma and Y. Ai, Sheathless inertial cell focusing and sorting with serial reverse wavy channel structures, Microsyst. Nanoeng., 2018, 4(1), 5.

46 G. S. A. Segré, Radial particle displacements in Poiseuille flow of suspensions, Nature, 1961, 189, 209-210. 
47 E. S. Asmolov, The inertial lift on a spherical particle in a plane Poiseuille flow at large channel Reynolds number, J. Fluid Mech., 1999, 381, 63-87.

48 D. Di Carlo, J. F. Edd, K. J. Humphry, H. A. Stone and M. Toner, Particle segregation and dynamics in confined flows, Phys. Rev. Lett., 2009, $102(9), 094503$.

49 T. L. Simon, Blood, Principles and Practice of Hematology, Transfusion, 2004, 44(2), 305-306.

50 D. Yang, G. Subramanian, J. Duan, S. Gao, L. Bai, R. Chandramohanadas and Y. Ai, A portable image-based cytometer for rapid malaria detection and quantification, PLoS One, 2017, 12(6), e0179161.

51 K. Mutafopulos, P. Spink, C. D. Lofstrom, P. J. Lu, H. Lu, J. C. Sharpe, T. Franke and D. A. Weitz, Traveling surface acoustic wave (TSAW) microfluidic fluorescence activated cell sorter ( $\mu$ FACS), Lab Chip, 2019, 19, 2435-2443.

52 E. Ozkumur, A. M. Shah, J. C. Ciciliano, B. L. Emmink, D. T. Miyamoto, E. Brachtel, M. Yu, P. I. Chen, B. Morgan, J. Trautwein, A. Kimura, S. Sengupta, S. L. Stott, N. M. Karabacak, T. A. Barber, J. R. Walsh, K. Smith, P. S. Spuhler, J. P. Sullivan, R. J. Lee, D. T. Ting, X. Luo, A. T. Shaw, A. Bardia, L. V. Sequist, D. N. Louis, S. Maheswaran, R. Kapur, D. A. Haber and M. Toner, Inertial focusing for tumor antigen-dependent and -independent sorting of rare circulating tumor cells, Sci. Transl. Med., 2013, 5(179), 179 ra47.
53 M. Mizuno, M. Yamada, R. Mitamura, K. Ike, K. Toyama and M. Seki, Magnetophoresis-integrated hydrodynamic filtration system for size- and surface marker-based twodimensional cell sorting, Anal. Chem., 2013, 85(16), 76667673.

54 B. N. G. Sajay, C.-P. Chang, H. Ahmad, P. Khuntontong, C. C. Wong, Z. Wang, P. D. Puiu, R. Soo and A. R. A. Rahman, Microfluidic platform for negative enrichment of circulating tumor cells, Biomed. Microdevices, 2014, 16(4), 537-548.

55 F. Del Giudice, H. Madadi, M. M. Villone, G. D'Avino, A. M. Cusano, R. Vecchione, M. Ventre, P. L. Maffettone and P. A. Netti, Magnetophoresis 'meets' viscoelasticity: deterministic separation of magnetic particles in a modular microfluidic device, Lab Chip, 2015, 15(8), 19121922.

56 C. Sunghwan and C. Young-Ho, in Continuous blood cell separation using a dielectrophoretic virtual pillar array, 2008 3rd IEEE International Conference on Nano/Micro Engineered and Molecular Systems, 6-9 Jan. 2008, pp. 974977.

57 H. S. Moon, K. Kwon, S. I. Kim, H. Han, J. Sohn, S. Lee and H. I. Jung, Continuous separation of breast cancer cells from blood samples using multi-orifice flow fractionation (MOFF) and dielectrophoresis (DEP), Lab Chip, 2011, 11(6), 11181125. 